\title{
Far field scattering pattern of differently structured butterfly scales
}

\author{
M. A. Giraldo $\cdot$ S. Yoshioka $\cdot$ D. G. Stavenga
}

Received: 16 July 2007 / Revised: 17 August 2007 / Accepted: 25 August 2007 / Published online: 20 December 2007

(C) The Author(s) 2007

\begin{abstract}
The angular and spectral reflectance of single scales of five different butterfly species was measured and related to the scale anatomy. The scales of the pierids Pieris rapae and Delias nigrina scatter white light randomly, in close agreement with Lambert's cosine law, which can be well understood from the randomly organized beads on the scale crossribs. The reflectance of the iridescent blue scales of Morpho aega is determined by multilayer structures in the scale ridges, causing diffraction in approximately a plane. The purple scales in the dorsal wing tips of the male Colotis regina act similarly as the Morpho scale in the blue, due to multilayers in the ridges, but the scattering in the red occurs as in the Pieris scale, because the scales contain beads with pigment that does not absorb in the red wavelength range. The green-yellow scales of Urania fulgens backscatter light in a narrow spatial angle, because of a multilayer structure in the scale body.
\end{abstract}

Keywords Single butterfly scale $\cdot$ Structural colour . Lambertian reflector $\cdot$ Scattering $\cdot$ Angular reflectance

\section{Introduction}

Many butterflies have vivid, colourful wing patterns, created by rows of partly overlapping scales with intricate

M. A. Giraldo $(\bowtie) \cdot$ D. G. Stavenga

Department of Neurobiophysics,

University of Groningen, Nijenborgh 4,

9747 AG Groningen, The Netherlands

e-mail: M.A.Giraldo@rug.nl

S. Yoshioka

Graduate School of Frontier Biosciences,

Osaka University, Suita, Osaka 565-0871, Japan spatial structures that scatter incident light. The scattered light can interfere coherently or incoherently. When the scale structures have spatial periodicity, the interference of coherent light waves often results in striking iridescences. The displayed colours are then called structural or physical. Without periodicity, light scattering is incoherent or random, resulting in white scales, unless they contain pigment that selectively absorbs in a certain wavelength range. In the latter case a pigmentary or chemical colour results (Fox and Vevers 1960; Vukusic and Sambles 2003; Kinoshita and Yoshioka 2005). Many butterfly species feature structural as well as pigmentary colours (Rutowski et al. 2005; Yoshioka and Kinoshita 2006a).

Each butterfly wing scale is the cuticular product of a single cell, with a rather flat, unstructured lower scale lamina and a highly structured upper lamina, typically consisting of longitudinal ridges connected by crossribs (Ghiradella 1998). The fine structure of butterfly scales is highly variable (Vukusic et al. 2000). For instance, the crossribs of the white scales of pierids are adorned with granules (Yagi 1954; Giraldo and Stavenga 2007; Morehouse et al. 2007), the ridges of Morpho scales and many male Pieridae are elaborated into multilayer structures (Ghiradella et al. 1972; Vukusic et al. 1999; Kinoshita et al. 2002), and many papilionids and lycaenids have scales with photonic crystal properties (Vukusic et al. 2002; Vukusic and Sambles 2003; Kertesz et al. 2006).

The scales reflect only part of the incident light flux, and the remaining part is transmitted unless it is absorbed by pigment. Because the scales are arranged in layers on both sides of the wing, incident light suffers reflection and transmission in each layer of the scale stacks, and therefore the wing reflectance is not solely due to backscattering by the top layer, the cover scales, but it is the cumulative effect of the scale stacks on both wing sides. Yoshioka and 
Kinoshita (2006b) investigated this phenomenon in Morpho wings, and to explain the reflectance spectra of intact wings from the spectra of individual scales they used a simplified scale stack model, assuming that normally incident light rays are also reflected and transmitted normally. Stavenga et al. (2006) developed a more general model for the reflectance of butterfly wings to interpret reflectance measurements performed on intact as well as partly or completely denuded wings of the small white butterfly, Pieris rapae. The basic assumption of the latter model, that the scales scatter randomly, was however not specifically demonstrated.

Knowledge of the spatial and spectral reflectance characteristics of single scales is essential to further develop our understanding of the coloration principles applied by butterflies. In the present study we investigate the scattering properties of wing scales of a variety of butterflies, and we correlate the scattering diagrams with the electron micrographs of the scale structure. We show that white scales of pierid butterflies are approximately random scatterers. Iridescent scales, with multilayer structures, exhibit directional reflection. Whereas some butterfly species appear to have scales with dominant iridescence, other species combine iridescence and scattering properties.

\section{Materials and methods}

\section{Animals}

We investigated the scales of a variety of butterflies. The small white, P. rapae, was obtained from a continuous culture maintained by Dr J. J. van Loon, Entomology Department, University of Wageningen (the Netherlands). The black jezebel, Delias nigrina, was captured near Bateman's Bay, Australia. The Morpho aega was purchased. The purple tip, Colotis regina, was received from the butterfly collection of the Royal Museum for Central Africa, Brussels (Belgium; curator Dr U. Dall'Asta). The moth Urania fulgens was a gift from Dr Marta Wolff, Entomology group, University of Antioquia (Medellín, Colombia).

Angular distribution of scattering by single scales

Single scales were isolated by gently pressing the wing of a butterfly on a glass plate. Subsequently, an isolated scale was glued to the tip of a glass micropipette (tip diameter ca. $5 \mu \mathrm{m})$. The micropipette was then mounted on a micromanipulator in the optical setup shown in Fig. 1a. The scale was always hanging with the longitudinal ridges of the scale oriented vertically. Light from a xenon lamp was focused on a pinhole with diameter $50 \mu \mathrm{m}$, which was

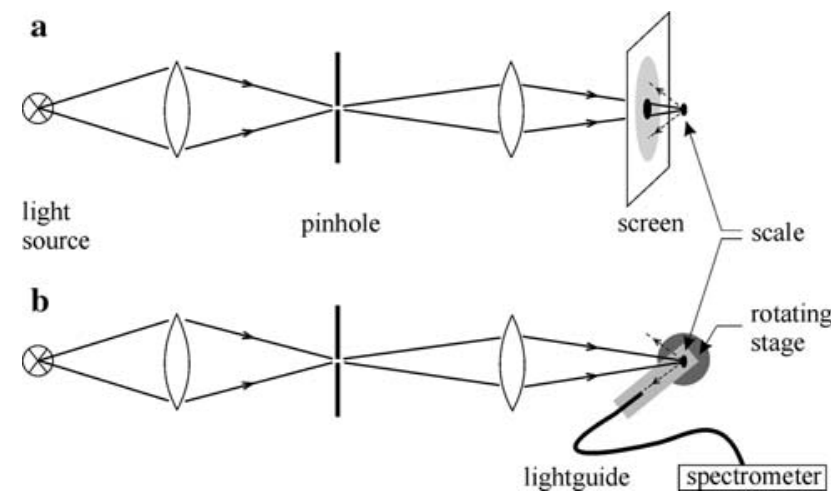

Fig. 1 Diagram of the optical system used for measuring the angular distribution of the scale reflectance. Light from a light source was focused on a pinhole, which was imaged on the scale. a A white screen with a small hole was placed in between the imaging lens and the scale. The light reflected by the scale caused a light pattern on the screen, which was photographed. b The light reflected by the scale was collected by a lightguide, which relayed the captured light to a spectrometer. The lightguide was mounted on a motorized stage rotating in the horizontal plane (top view drawing)

subsequently imaged on the scale. The light beam, which had an aperture of $<10^{\circ}$, passed a small hole in a screen before it reached the scale. The scale reflected (that is, back-scattered) part of the incident light. The angular spread of the reflected light was documented by photographing the light distribution at the white backside of the screen (Fig. 1a). We used a Nikon Coolpix 990 (Fig. 2) and an Olympus DP70 (Fig. 6). The digital images were processed with MatLab (Fig. 6).

\section{Spectrophotometry}

The angular dependence of the light scattering by the scale was measured with a lightguide, which was mounted on a rotating motor and connected to a spectrometer (Yoshioka and Kinoshita 2006b). The scales were adjusted so that the scale plane was perpendicular to the light beam, as judged by the symmetrical reflection pattern with respect to the axial direction. The experiments were run using a Labview interface, which allowed the measurement of reflectance spectra in angular steps of $1^{\circ}$ over a $180^{\circ}$ angle (Fig. 1b). Angular reflectance curves were calculated for a series of wavelengths with $10 \mathrm{~nm}$ interval by sequentially averaging the reflectance spectra over $10 \mathrm{~nm}$ wavelength ranges. In addition, the spectral reflectances of single scales were measured with a microspectrophotometer, consisting of a xenon light source, a Leitz Ortholux microscope, and a fiber optic spectrometer. The microscope objective was an Olympus $20 \times$, NA 0.46 . A white reflectance standard (Spectralon, Labsphere, North Sutton, NH, USA) served as the reference in all cases. 

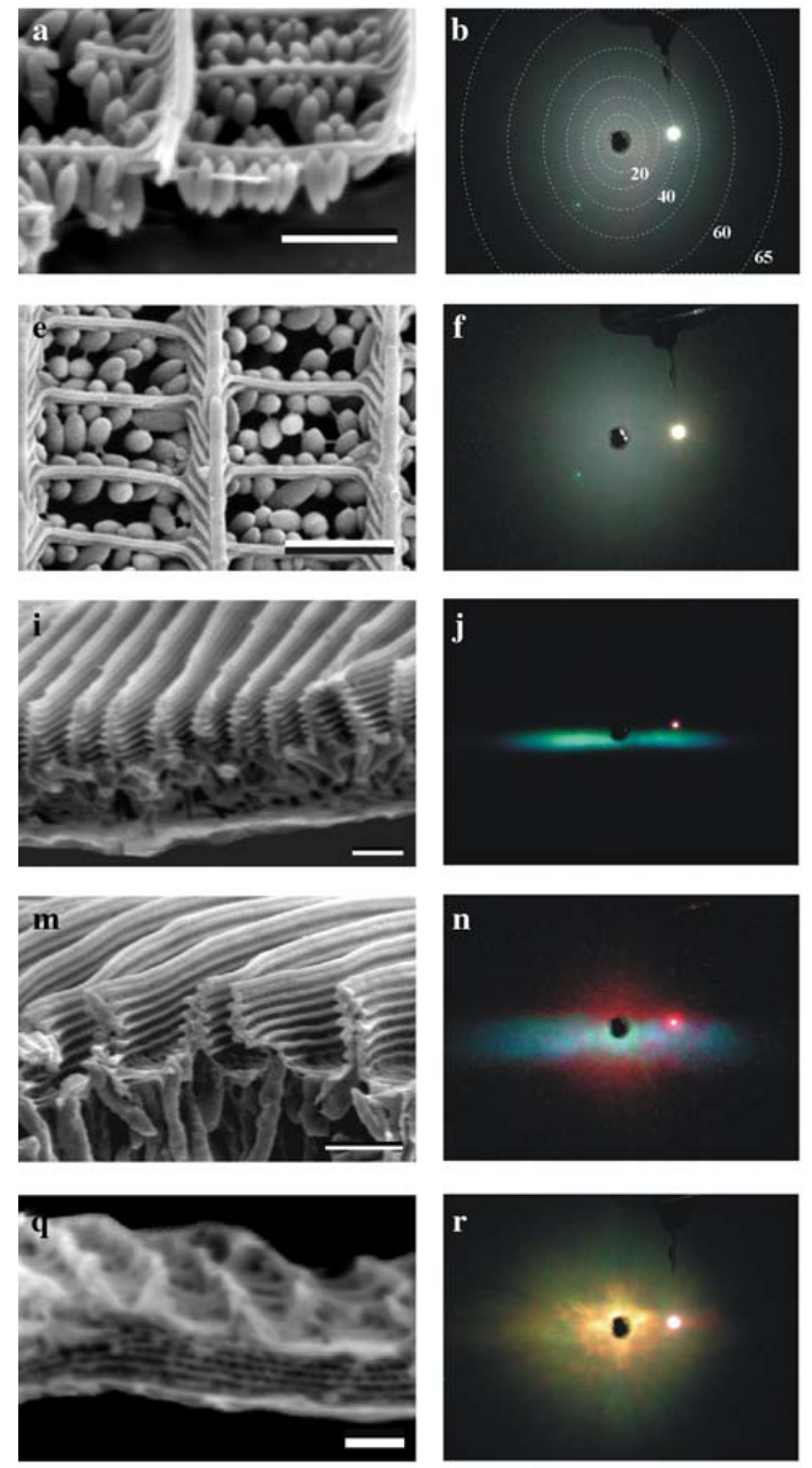

Fig. 2 Angular and spectral reflectance of isolated butterfly scales. a-d White scale of the dorsal forewing of a male Pieris rapae. $\mathbf{e}-\mathbf{h}$ White scale from the dorsal forewing of a male black jezebel, Delias nigrina. i-l Blue scale of a Morpho aega. $\mathbf{m}-\mathbf{p}$ Purple scale from the tip of the dorsal forewing of a male purple tip, Colotis regina. q-t Orange-white scale of Urania fulgens. First column: scanning electron microscopy of scale cross-sections made by cutting pieces of wing with a razor blade. Bars $1 \mu \mathrm{m}$. Second column: photographs of the angular distribution of light reflected by a scale and subsequently reflected by a white screen, where the scale was illuminated with a narrow beam of white light (see

\section{Electron microscopy}

Subsequent to the microspectrophotometry, the single scales were prepared for scanning electron microscopy (SEM). Additionally, pieces of wing were cut and put on the SEM-holder in different positions to visualize the upper surface as well as cross-section of the scales.
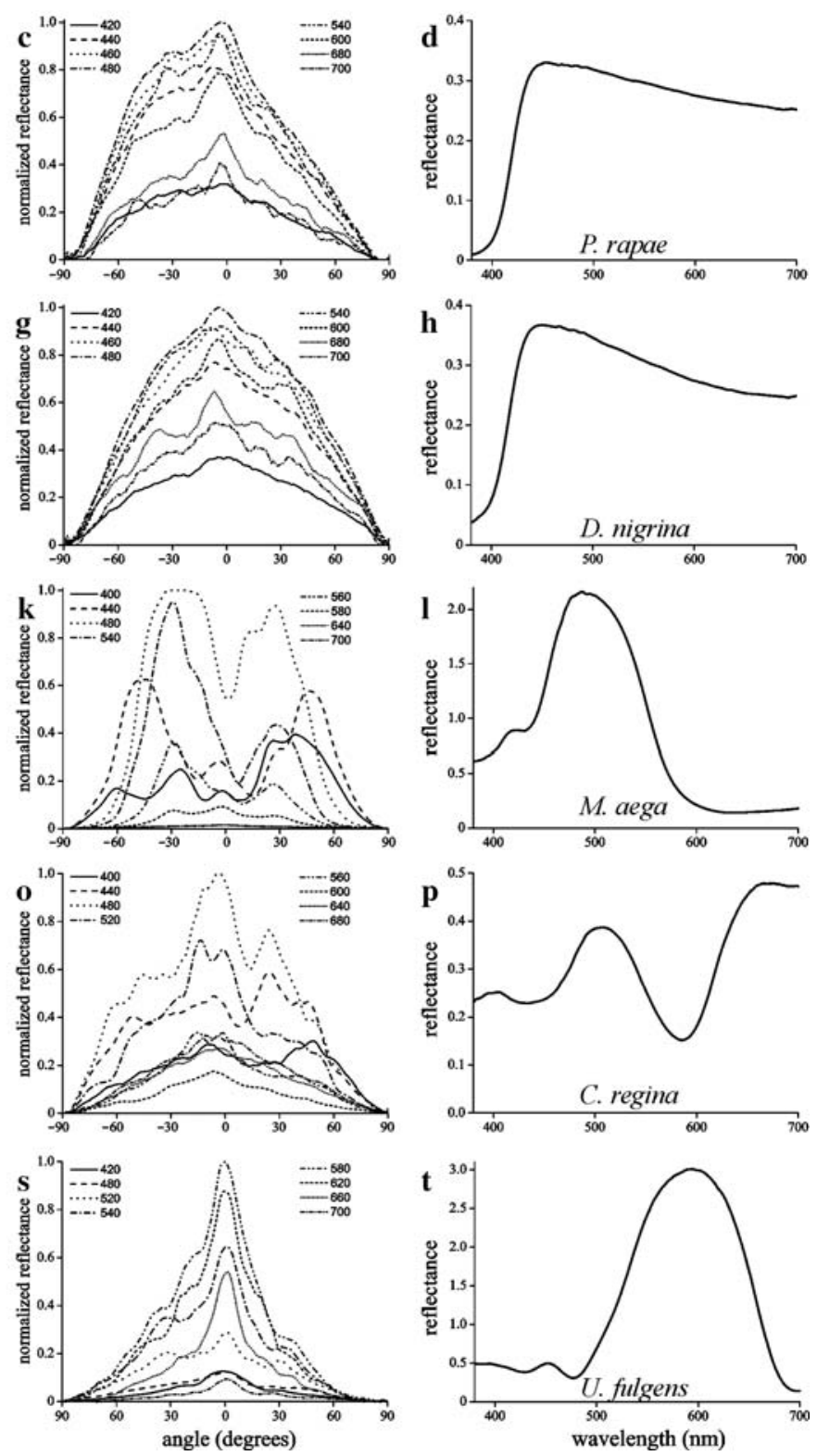

Fig. 1a). The scales were glued at the tip of a glass micropipette, which was suspended from above on a micromanipulator; see the black shadow in the upper part of $\mathbf{b}$ and $\mathbf{f}$. The bright spot at the right of the central black hole is due to light forward scattered by the scale. The ellipses in $\mathbf{b}$ (due to the slightly oblique position of the camera) represent directional angles in steps of $10^{\circ}$, from $10^{\circ}$ to $60^{\circ}$, as well as that for $65^{\circ}$. The hole in the screen is ca. $8^{\circ}$. Third column: angular dependence of the reflectance in the horizontal plane, measured with the setup of Fig. 1b. Fourth column: reflectance spectra of single scales measured with a microspectrophotometer

Samples were sputtered with palladium for 5 min with $800 \mathrm{~V}$ and 200 mTorr (Hummer, Alexandria, VA, USA). A Philips XL-30 scanning electron microscope with a voltage of $3 \mathrm{kV}$ was used to investigate the scale anatomy. For transmission electron microscopy, wing pieces were prepared as usual. In brief, samples were embedded in agar for better handling, prefixed in $2 \%$ glutaraldehyde in 
$0.1 \mathrm{M}$ sodium cacodylate buffer, and postfixed in $1 \%$ $\mathrm{OsO}_{4} / 1.5 \% \mathrm{~K}_{4} \mathrm{Fe}(\mathrm{CN})_{6}$ in $0.1 \mathrm{M}$ cacodylate. Subsequent washing with double-distilled water and dehydration with an alcohol series that ended with $100 \%$, were followed by propylene oxide during $30 \mathrm{~min}$ and embedding in Epon. Post-microtomed samples were contrasted with uranyl acetate in methanol for $2 \mathrm{~min}$ and lead in water for $1 \mathrm{~min}$, and were then examined with a Philips 201 transmission electron microscope.

\section{Results}

The white scales of the small white, P. rapae, are marked by ovoid beads that adorn the crossribs (Fig. 2a). The angular light scattering of a single scale can be readily visualized with the setup of Fig. 1a, where a beam of white light is focused on a scale via a small hole in a screen. Figure $2 b$ is a photograph of the screen, showing the angular distribution of the light reflected by a white scale taken from the dorsal wing of a male small white, $P$. rapae. The scale scatters light approximately circular-symmetrically, suggesting that the scale acts as a diffuse scatterer.

The angular distribution of the scattering was quantitatively investigated with the setup of Fig. 1b, where the light reflected by the scale is measured as a function of angle in the horizontal plane. Figure $2 \mathrm{c}$ presents the reflectance of the scale of Fig. $2 b$ as a function of angle for a number of wavelengths, normalized to the maximal reflectance value; the angle is $0^{\circ}$ for the normal to the scale. The scale's reflectance spectrum for normally incident light is given in Fig. 2d. The reflectance is high in the visible wavelength range, but it is low in the ultraviolet, because of an ultraviolet-absorbing pigment, presumably leucopterin (Wijnen et al. 2007), which is concentrated in pigment granules, the ovoid beads (Fig. 2a). The beads act as strong scatterers at wavelengths where the pigment absorption is negligible (Stavenga et al. 2004; Giraldo and Stavenga 2007; Morehouse et al. 2007). In addition to the beads, the other structures of the scale, that is the ridges and crossribs of the upper lamina of the scale as well as the lower lamina, contribute to the scattering (Fig. 2c).

Figure $2 \mathrm{e}-\mathrm{h}$ presents a similar set of data for a white scale of the dorsal forewing of a male black jezebel, $D$. nigrina. This case is obviously very similar to that of the white $P$. rapae scale. The scale anatomy with crossribs studded with beads is very similar (Fig. 2e), the scattering is again approximately random (Fig. 2f, g), and the reflectance spectrum is also high in the visible and low in the UV (Fig. 2h).

The strikingly blue $M$. aega has scales where the lamellae of the ridges form multilayers (Fig. 2i). Different from Morpho didius, for example, which has glass cover scales and strongly pigmented ground scales (Vukusic et al. 1999), cover and ground scales of M. aega cannot be distinguished. The scattering diagram of a scale of a M. aega is a horizontal stripe (Fig. 2j), perpendicular to the vertically oriented scale ridges. The angular dependence of the reflectance varies strongly with the wavelength (Fig. 2k), and the reflectance measured with normally incident light features a distinct peak in the blue (Fig. 21), which is due to the multilayered structure of the scale ridges (Fig. 2i; see also Vukusic et al. 1999; Kinoshita et al. 2002; Yoshioka and Kinoshita 2004). A reflectance peak value of more than two results, because the scale's scattering is highly directional and the reflectance was measured relative to the diffusely scattering white standard (Fig. 21).

The purple scales at the dorsal wing tips of the male purple tip, $C$. regina, have ridges that are fine-structured similarly as in the case of M. aega (Fig. $2 \mathrm{~m}$ ). The purple scale features a scattering diagram with a blue stripe and a red diffuse pattern (Fig. 2n), which is a mixture of the stripe phenomenon of Fig. $2 j$ and the diffuse patterns of Fig. $2 b, f$. The blue stripe is due to light backscattered by the finestructured ridges, and the red diffuse pattern results from randomly scattered light, filtered by a pigment contained in granules below the multilayered ridges (Fig. $2 \mathrm{~m}$ ). The blue peaking reflectance spectrum, shown in Fig. 2p, is mainly due to reflection by the ridges, and the red band, above $600 \mathrm{~nm}$, is mainly caused by the light scattering pigment granules (Fig. 2p).

A green-yellow reflecting scale of the moth $U$. fulgens has between the upper and lower scale laminae (Fig. 2q) an elaborate multilayer system, which yields a spatially restricted, directional scattering diagram (Fig. 2r, s). The high amplitude of three of its reflectance spectrum is again due to the directionality of the scale reflectance (Fig. 2t). The reflectance spectrum features a distinct band, peaking at $590 \mathrm{~nm}$, with halfwidth about $120 \mathrm{~nm}$, indicating that an interference reflector is involved.

To evaluate whether the beaded scales of $P$. rapae, $D$. nigrina and C. regina act as diffuse scatterers, the angular reflectances of these species were normalized and compared with a Lambertian reflector (Fig. 3). The wavelengths selected are outside the absorption bands of the pigments, which means that the wavelengths chosen for $P$. rapae and $D$. nigrina are in the visible spectrum, but for $C$. regina only in the red wavelength range. The white scale of D. nigrina (Fig. 3b) well approximates a Lambertian reflector. The angular distribution patterns of the white scale of P. rapae slightly deviate from the ideal curve (Fig. 3a), but notably the purple scale of $C$. regina is not a perfectly diffused red scatterer. Presumably this is due to the highly structured, multilayered ridges. 

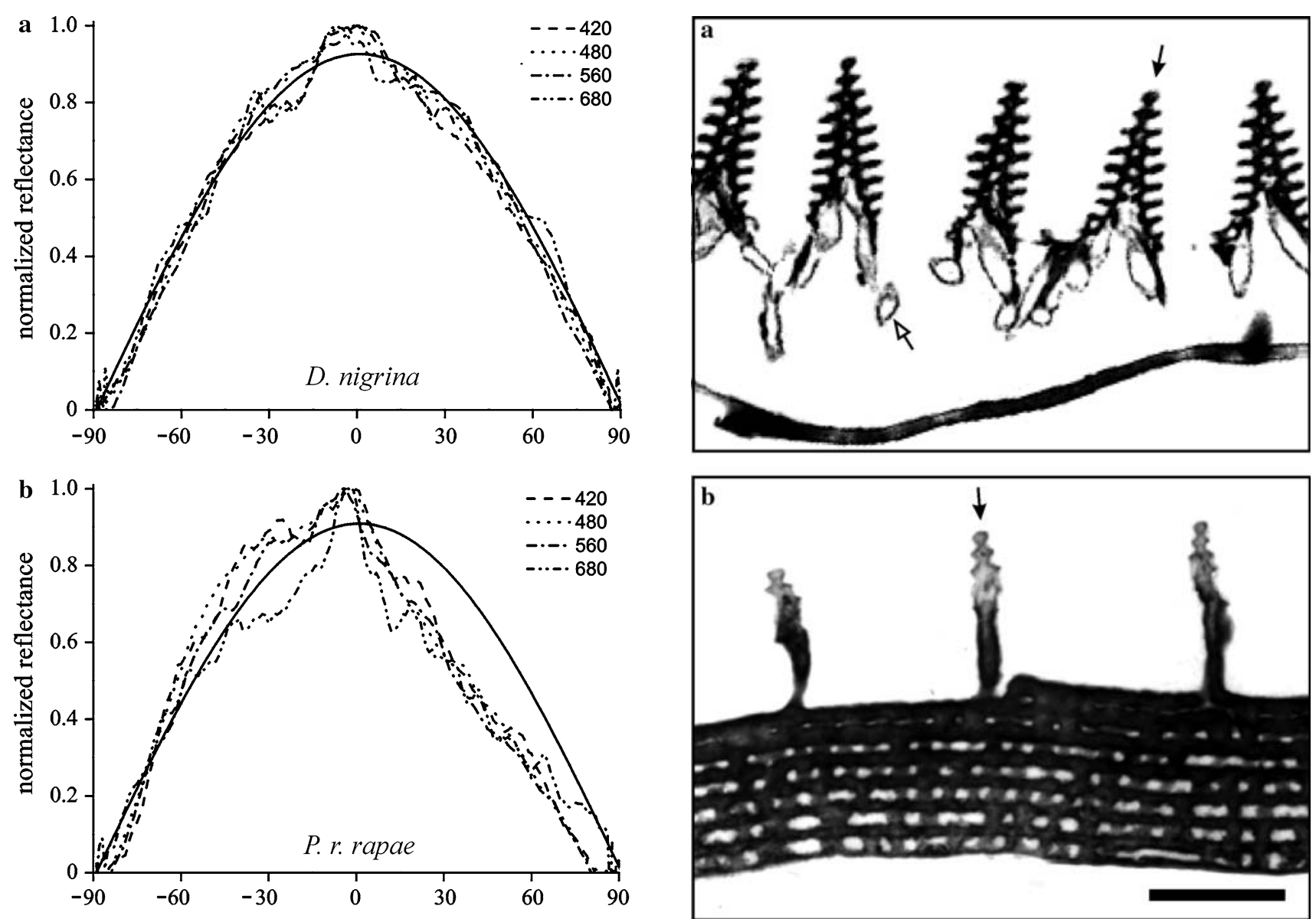

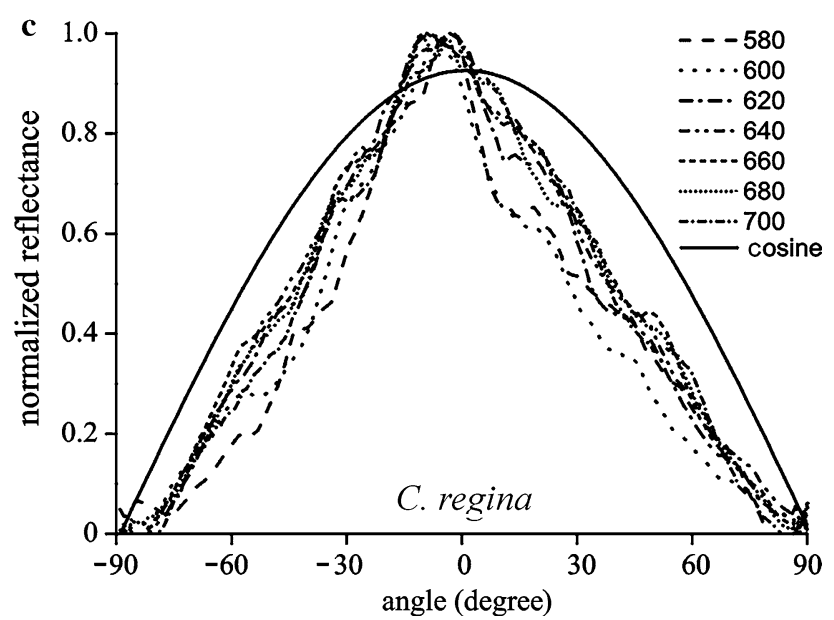

Fig. 3 Normalized angular reflectances measured from the scales of the three pierid butterflies, $P$. rapae, D. nigrina, and $C$. regina, compared to a Lambertian reflector (bold line). Angular reflectance curves are shown in the wavelength range where the reflectance is high, that is, where pigment absorption is low. Four wavelengths covering the visible range are shown for $P$. rapae (a) and D. nigrina (b). The scattering diagrams of $D$. nigrina well approximate a Lambertian reflector, but the angular dependence of the scattering by $C$. regina shows regular peaks, which indicates that the light scattered by the pigment granules is affected by the multilayered ridges
Fig. 4 Transmission electron microscopy images of a cover scale of the dorsal wing of a male brimstone, Gonopteryx rhamni (a), and of a scale of Urania fulgens (b). Ridges are indicated by closed arrows. The brimstone scale has multilayers restricted to the ridges. The space between ridges and crossribs is studded with pigment granules (open arrow). U. fulgens has continuous multilayers connected by numerous pillars that result in spreading of the scattering. Bar: $1 \mu \mathrm{m}$

\section{Discussion}

The relationship between the optical properties of butterfly scales and their structure has been the topic of several studies (e.g., Vukusic et al. 1999, 2002; Kinoshita et al. 2002). Most of the previous investigations have focused on iridescent scales. Here, we have compared the reflection pattern of five differently structured single butterfly scales that scatter light coherently or incoherently, or both.

We started with a simple white scale common to many species of the pierid subfamily Pierinae. Due to the characteristic beaded structure light scattering is strong, thus causing the intense white colour. We find that the white pierid scales approximate the properties of a Lambertian diffuser, 
Fig. 5 Scattering by scales of Morpho aega. a Single scale about normally illuminated by a beam with about $50 \mu \mathrm{m}$ diameter; the outline of the scale is visible because of an additional, obliquely illuminating beam (bar: $50 \mu \mathrm{m}$ ). b Far field scattering pattern of the single scale photographed at a white screen (see Fig. 2j). The bright white spot is due to light scattered at the hole in the white screen and direct scattering by the scale. The interrupted circles indicate the angular directions of the scattering in steps of $10^{\circ}$. c A wing piece about normally illuminated by a beam with about $900 \mu \mathrm{m}$ diameter; cover and ground scales cannot be distinguished (bar: $200 \mu \mathrm{m}$ ). d Far field scattering pattern of the wing piece. The white spot is due to light scattered at the hole in the white screen and to direct scattering by the scale
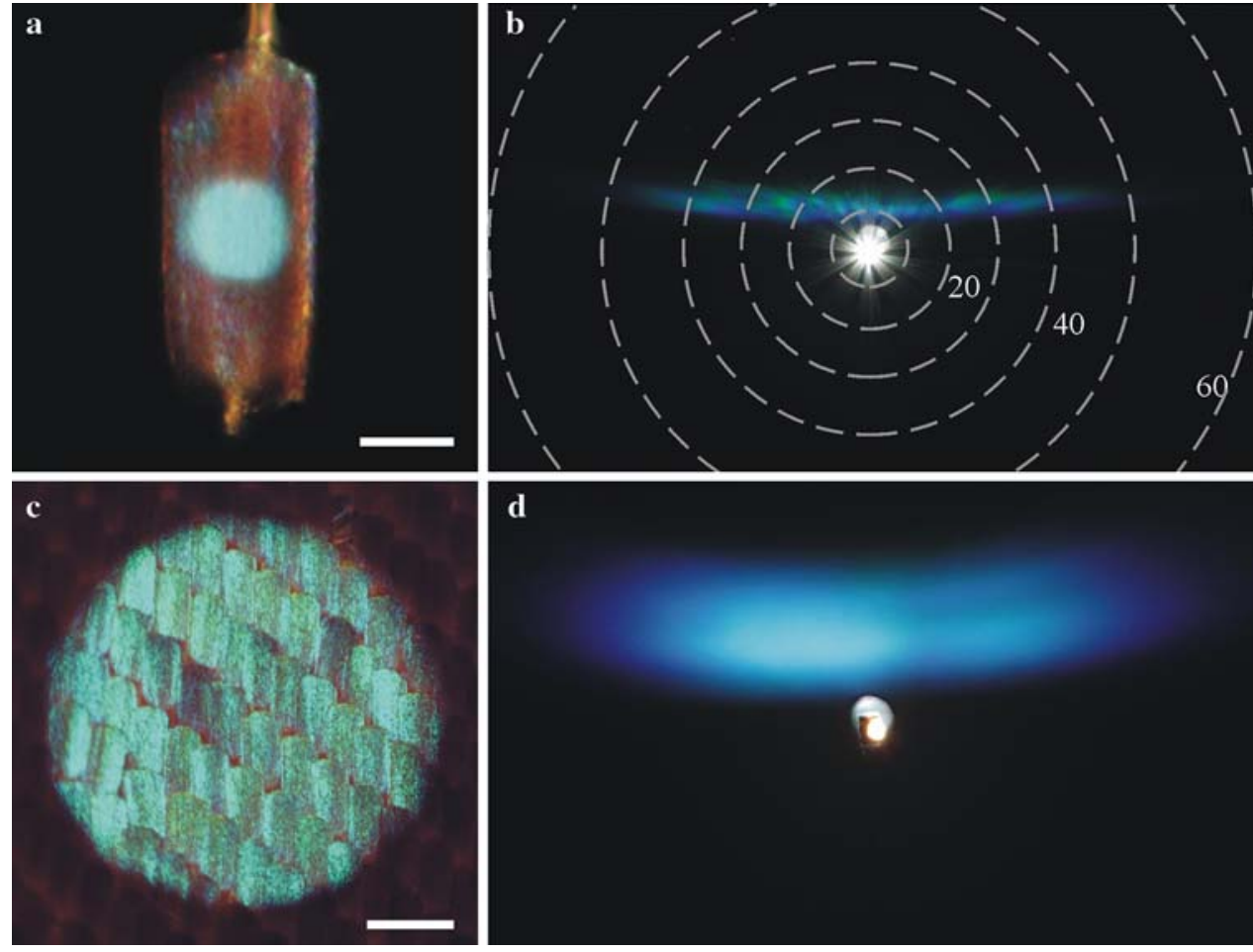

d

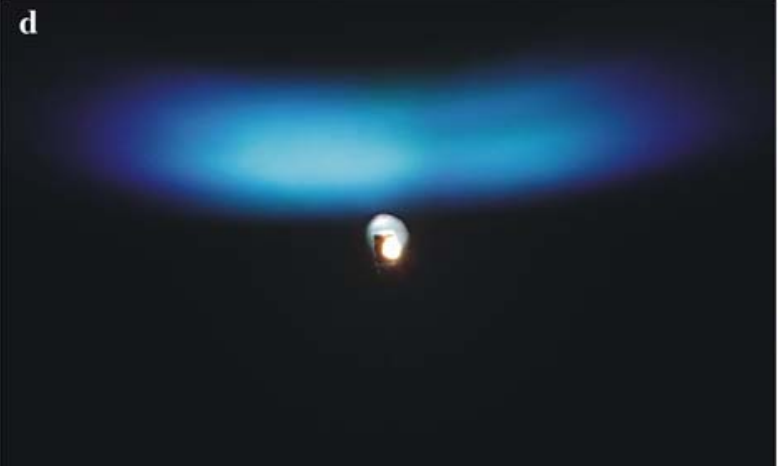

at least in the wavelength range where pigment absorption is negligible (Fig. 3).

The ridges of the scales of Morpho butterflies cause a blue colour. Melanin pigment below the multilayered ridges absorbs stray light over the whole visible wavelength range, including the ultraviolet, thus supporting the strikingly blue wing colour (Yoshioka and Kinoshita 2006a). The pigment of the scales in the dorsal wing tips of C. regina also absorbs stray light, but not in the long-wavelength range. The remaining red light together with the blue iridescence causes the purple colour.

A similar, however, short-wavelength-shifted case is formed by the yellow scales of the dorsal wings of many male butterflies of the pierid subfamily Coliadinae, where a UV and blue absorbing pigment is combined with UV iridescence (Ghiradella et al. 1972; Rutowski et al. 2005). Figure 4a is a transmission electron micrograph of a yellow cover scale from the dorsal wing of a male brimstone, Gonepteryx rhamni. The UV iridescent, yellow scales of the male brimstone have numerous beads, which contain the yellow pigment xanthopterin (Wijnen et al. 2007). The beads are seen in Fig. 4a as empty ovoids, but this is presumably due to the procedures for transmission electron microscopy (Morehouse et al. 2007).

The scattering diagram of $U$. fulgens is not perfectly directional (Figs. 2r, s), which should have been the case when the scales consisted of an ideal multilayer. Transmission electron microscopy shows that between the multilayers exist pillars (Fig. 4b), which presumably cause the spread in the scattering diagram. Of course, the scales-and therefore the multilayers - are also not perfectly flat. The ridges (see Figs. 2q, 4b) will further contribute to some diffuse scattering.

Knowing how light is scattered by individual scales is necessary for a proper understanding of butterfly wing coloration. As has been previously demonstrated, wing reflectance is a cumulative effect due to multiple reflection and transmission by the layers of scales on the butterfly wing (Yoshioka and Kinoshita 2006b; Stavenga et al. 2006). The scales are often arranged in quite regular rows and have approximately the same orientation with respect to the wing surface, but of course there are deviations from perfect order. This will be rather irrelevant in the case of the small white, $P$. rapae, where scattering is approximately diffuse, but in cases where scattering is highly directional, as in Morpho butterflies, disordered scale arrangement will result in broadening of the scattering diagrams. This is illustrated in Fig. 5, where scattering by a single scale of $M$. aega (Fig. 5a) is compared with scattering by an array of scales on the wing (Fig. 5c). The far-field scattering diagram of a single scale is a narrow stripe (Fig. 5b; see also Fig. 2j), but that of a set of scales is a distinctly broadened stripe (Fig. 5d). The angular extent of the scattering by the single scale in the vertical plane is $<10^{\circ}$, but that of the scale set is about $30^{\circ}$ (Y, Fig. 6). The broadening of about $20^{\circ}$ will be due to a rotational variation of the scale plane around the long axis of the scales. The tilt angle, that is the angle of the scale plane with the wing, will have a similar variation, as the scattering diagram of the scale set is about $20^{\circ}$ wider 


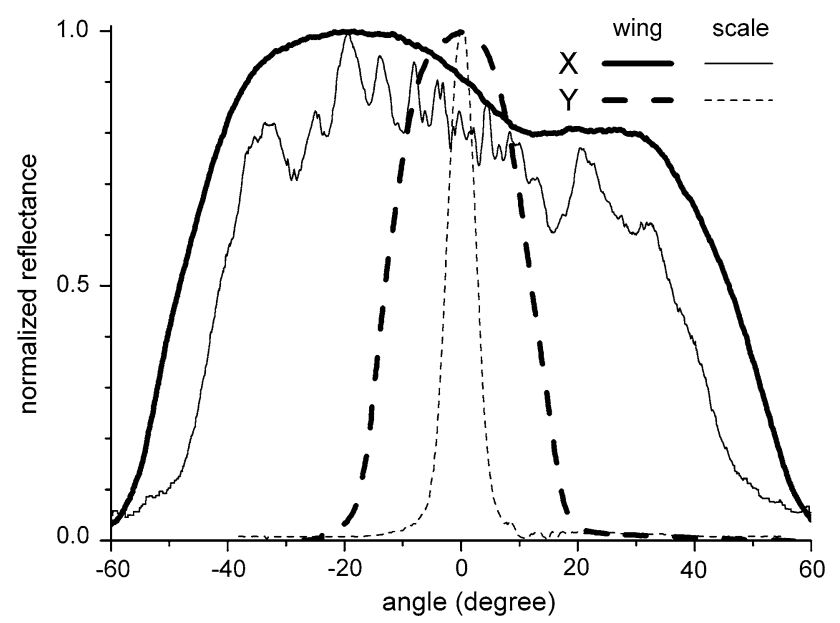

Fig. 6 Spatial profiles of the scattering diagrams of Fig. 5b, d. The extent of the scattering in the horizontal plane $(X)$ is much broader than the scattering in the vertical plane $(Y)$ for both the single scale as for the intact wing piece. The angular width in the vertical plane of the scattering by the single scale is $<10^{\circ}$, but the width in the horizontal plane is about $100^{\circ}$. The vertical angular extent of the scattering by the wing piece is about $30^{\circ}$, meaning a broadening of about $20^{\circ}$ with respect to the single scale, and the extent in the horizontal plane is similarly broadened

than that of the single scale (X, Fig. 6). Further broadening of the scattering diagram will occur when reflection by the complete wings of a Morpho butterfly is considered. The dynamic changes of light scattering by a Morpho flying in a natural environment, and how the butterflies will be perceived by conspecifics and predators will be interesting themes for future research.

Acknowledgments Prof. J. T. M. de Hosson and G. ten Brink (Materials Science Department, University of Groningen) provided essential facilities for scanning electron microscopy, and Dr. H. van der Want together with D. Kalicharan and H. Blaauw (Electron Microscopy Department, Cellular Biology, University of Groningen) provided the support for the transmission electron microscopy. We thank Dr. H. Ghiradella for reading the manuscript and for valuable suggestions. A grant from the University of Osaka, Japan, enabled the research stay of M. A. G. in the Graduate School of Frontier Biosciences in Osaka. A visit to the Royal Museum for Central Africa, Brussels, was supported by the EU via SYNTHESYS. Further financial support was given by the EOARD (Grant 063027).

Open Access This article is distributed under the terms of the Creative Commons Attribution Noncommercial License which permits any noncommercial use, distribution, and reproduction in any medium, provided the original author(s) and source are credited.

\section{References}

Fox HM, Vevers G (1960) The nature of animal colours. Sidgwick \& Jackson, London
Ghiradella H (1998) Hairs, bristles, and scales. In: Locke M (ed) Microscopic anatomy of invertebrates. Insecta, vol 11A. WileyLiss, New York, pp 257-287

Ghiradella H, Aneshansley D, Eisner T, Silberglied R, Hinton HE (1972) Ultraviolet reflection of a male butterfly: interference color caused by thin-layer elaboration of wing scales. Science 178:1214-1217

Giraldo MA, Stavenga DG (2007) Sexual dichroism and pigment localization in the wing scales of Pieris rapae butterflies. Proc R Soc B 274:97-102

Kertész K, Bálint Z, Vértesy Z, Márk GI, Lousse V, Vigneron JP, Biró LP (2006) Photonic crystal type structures of biological origin: structural and spectral characterization. Curr Appl Phys 6:252258

Kinoshita S, Yoshioka S (2005) Structural colors in nature: the role of regularity and irregularity in the structure. ChemPhysChem $6: 1-19$

Kinoshita S, Yoshioka S, Kawagoe K (2002) Mechanisms of structural colour in the Morpho butterfly: cooperation of regularity and irregularity in an iridescent scale. Proc R Soc Lond B 269:14171421

Morehouse NI, Vukusic P, Rutowski R (2007) Pterin pigment granules are responsible for both broadband light scattering and wavelength selective absorption in the wing scales of pierid butterflies. Proc R Soc B 274:359-366

Rutowski RL, Macedonia JM, Morehouse N, Taylor-Taft L (2005) Pterin pigments amplify iridescent ultraviolet signal in males of the orange sulphur butterfly, Colias eurytheme. Proc R Soc B 272:2329-2335

Stavenga DG, Stowe S, Siebke K, Zeil J, Arikawa K (2004) Butterfly wing colours: scale beads make white pierid wings brighter. Proc R Soc Lond B 271:1577-1584

Stavenga DG, Giraldo MA, Hoenders BJ (2006) Reflectance and transmittance of light scattering scales stacked on the wings of pierid butterflies. Opt Express 14:4880-4890

Vukusic P, Sambles JR (2003) Photonic structures in biology. Nature 424:852-855

Vukusic P, Sambles JR, Lawrence CR, Wootton RJ (1999) Quantified interference and diffraction in single Morpho butterfly scales. Proc R Soc Lond B 266:1403-1411

Vukusic P, Sambles JR, Ghiradella H (2000) Optical classification of microstructure in butterfly wing-scales. Photonics Sci News 6:61-66

Vukusic P, Sambles JR, Lawrence CR, Wootton RJ (2002) Limitedview iridescence in the butterfly Ancyluris meliboeus. Proc R Soc Lond B 269:7-14

Wijnen B, Leertouwer HL, Stavenga DG (2007) Colors and pterin pigmentation of pierid butterfly wings. J Insect Physiol 53:12061217

Yagi N (1954) Note of electron microscope research on pterin pigment in the scales of pierid butterflies. Annot Zool Jpn 27:113-114

Yoshioka S, Kinoshita S (2004) Wavelength-selective and anisotropic light-diffusing scale on the wing of the Morpho butterfly. Proc R Soc B 271:581-587

Yoshioka S, Kinoshita S (2006a) Structural or pigmentary? Origin of the distinctive white stripe on the blue wing of a Morpho butterfly. Proc R Soc B 273:129-134

Yoshioka S, Kinoshita S (2006b) Single-scale spectroscopy of structurally colored butterflies: measurements of quantified reflectance and transmittance. J Opt Soc Am A 23:134-141 